\title{
Numerical modelling of drifting flame tubes
}

\author{
R. W. Thatcher ${ }^{1} \quad$ R. O. Weber ${ }^{2}$
}

(Received 4 August 2006; revised 12 December 2007)

\begin{abstract}
Stationary, isolated flame tube and stationary, multiple flame tubes are found both experimentally and numerically in a counterflow burner. For some flames established experimentally, moving multiple flame tubes are observed. We examine numerically the effect of placing two isolated flame tubes in close proximity to each other. We find that the influence of one flame tube on the other is to cause the tubes to move and drift apart.
\end{abstract}

\section{Contents}

\section{Introduction}

2 The governing equations

See http://anziamj.austms.org.au/ojs/index.php/ANZIAMJ/article/view/71 for this article, (c) Austral. Mathematical Soc. 2007. Published December 19, 2007. ISSN 1446-8735 
3 Numerical method and grid definition

C623

4 Numerical results for two flame tubes

C625

5 Conclusion

C627

References

C627

\section{Introduction}

The ultimate aim of this joint project was to model numerically the drifting apart of flame balls in micro-gravity. Some remarkable observations have been recorded in micro-gravity experiments pioneered by Ronney [4]. These experiments revealed that when several flame balls were created simultaneously, they very slowly drift apart. As a first step in this project we investigate a two dimensional flame which has some similarities to three dimensional flame balls, namely flame tubes in a counterflow burner.

The existence of multiple and isolated, stationary flame tubes in a counterflow for certain fuel mixtures was shown numerically for non-premixed combustion [5] and was observed for premixed combustion [3]. The experiments also showed that flame tubes may drift in the combustion chamber. More recently, stationary flame tubes at unit Lewis number have been modelled in the presence of heat loss for premixed combustion [1]. Moreover, Daou et al. [1] also predicted moving, isolated flame tubes. Similar results for non-premixed flow were were also predicted by Daou et al. [2]. The two dimensional calculations described here consider numerically what happens when one flame tube is placed in close proximity to another in a counterflow.

We consider a simple chemical model of a premixed flame in a counterflow burner. A typical burner is illustrated schematically in Figure 1. A mixture of fuel and oxidant enter the burner through the injectors, gases exit the 

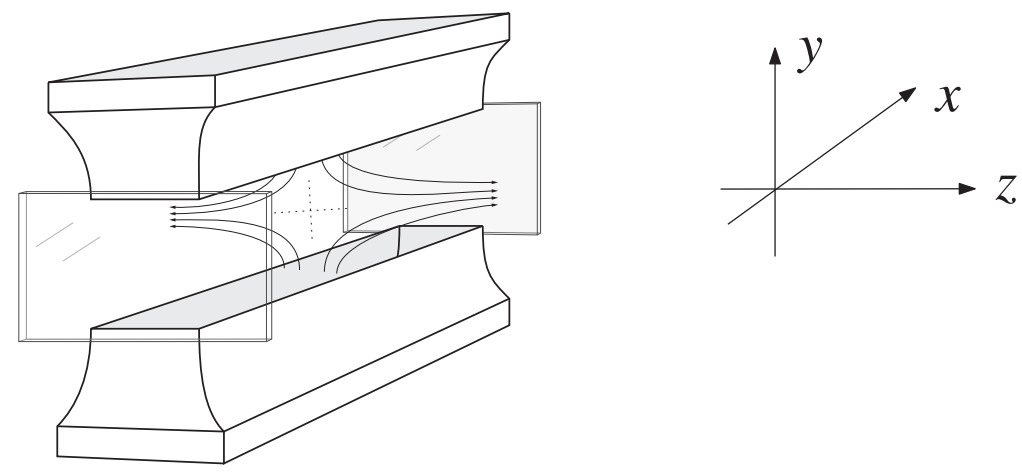

FiguRE 1: Schematic representation of a counterflow burner.

burner at the sides. In the mathematical model of a counterflow burner we choose the $x$-coordinate along the plates, the $y$-coordinate between the plates and the $z$-coordinate across the plates with origin in the centre of the burner. Thus gases enter from the $\pm y$ direction, exit in the $\pm z$ direction and there is no flow in the $x$ direction. When a spark is ignited in the burner a flame can be established. This flame lies close to the $y=0$ plane and, for some distance in the $z$ dimension close to the central plane $z=0$, is essentially independent of $z$. For most flames established in the burner the flame fills the burner in the $x$ dimension and is independent of $x$. Such a flame is essentially one dimensional, that is it depends only on $y$. For certain mixtures of fuel and for high enough strain rate a single cellular flame or multiple cellular flames become established in the burner rather than a single flame filling the burner. This flame, for small $z$, is independent of the $z$ dimension and is two dimensional depending on $x$ and $y$ coordinates only. It is called a flame tube. 


\section{The governing equations}

The combustion process is represented by the one-step irreversible reaction of the form

$$
\text { Fuel } \longrightarrow \text { Products }+ \text { Heat. }
$$

The reaction rate $\omega$ defined as the mass of fuel consumed per unit volume and unit time is assumed to follow the Arrhenius law of the form

$$
\omega=\rho \mathrm{Y}_{\mathrm{F}} B \exp (-E / R T),
$$

where for some constant $B$ the parameters $\rho, T, \mathrm{Y}_{\mathrm{F}}, E$ and $R$ represent respectively the density, the temperature, the mass fraction of the fuel, the activation energy and the universal gas number. We assume a constant density and that the heat capacity $c_{p}$, the thermal diffusivity $\mathrm{D}_{\mathrm{T}}$, and the diffusion coefficient $\mathrm{D}_{\mathrm{F}}$ are all constant. The velocity field in the counterflow burner has components $(0,-a y, a z)$, where $a$ is the strain rate. The general non-adiabatic time dependent equations for combustion on the central plane is independent of $z$ close to $z=0$ and are

$$
\begin{aligned}
\frac{\partial \mathrm{Y}_{\mathrm{F}}}{\partial t}-a y \frac{\partial \mathrm{Y}_{\mathrm{F}}}{\partial y} & =\mathrm{D}_{\mathrm{F}}\left(\frac{\partial^{2} \mathrm{Y}_{\mathrm{F}}}{\partial x^{2}}+\frac{\partial^{2} \mathrm{Y}_{\mathrm{F}}}{\partial y^{2}}\right)-\frac{\omega}{\rho}, \\
\frac{\partial T}{\partial t}-a y \frac{\partial T}{\partial y} & =\mathrm{D}_{\mathrm{T}}\left(\frac{\partial^{2} T}{\partial x^{2}}+\frac{\partial^{2} T}{\partial y^{2}}\right)+\frac{Q}{c_{p}} \frac{\omega}{\rho},
\end{aligned}
$$

where $T_{0}$ is the temperature of the unburnt gas and $Q$ the heat released per unit mass of fuel consumed in unit time. The temperature $T_{b}$ of the burnt gases is

$$
T_{b}=T_{0}+\frac{Q \mathrm{Y}_{\mathrm{F}_{0}}}{c_{p}},
$$

where $Y_{F_{0}}$ is the mass fraction of the fuel in the fresh mixture. We define the following rescaled variables:

$$
x^{\prime}=\frac{x}{L}, \quad y^{\prime}=\frac{y}{L}, \quad t^{\prime}=\frac{t}{t_{0}}, \quad F^{\prime}=\frac{\mathrm{Y}_{\mathrm{F}}}{\mathrm{Y}_{\mathrm{F}_{0}}}, \quad T^{\prime}=\frac{T-T_{0}}{\left(T_{b}-T_{0}\right)} .
$$


We choose $L$ as the thickness of the thermal mixing layer, thus

$$
L=\sqrt{2 \mathrm{D}_{\mathrm{T}} / a},
$$

and define the Zeldovich number as

$$
\beta=\frac{E}{R}\left(\frac{T_{b}-T_{0}}{T_{b}^{2}}\right)
$$

We define the Damköhler number $\delta$ so that $\delta \beta^{2}=\frac{2}{a} B \exp \left[-E /\left(R T_{b}\right)\right]$ and note that $\delta$ is inversely proportional to the strain rate $a$. Finally we choose $t_{0}$ so that $a t_{0}=2$ then

$$
\begin{aligned}
& \frac{\partial F^{\prime}}{\partial t^{\prime}}-2 y^{\prime} \frac{\partial F^{\prime}}{\partial y^{\prime}}=\frac{\mathrm{D}_{\mathrm{F}}}{\mathrm{D}_{\mathrm{T}}}\left(\frac{\partial^{2} F^{\prime}}{\partial x^{\prime 2}}+\frac{\partial^{2} F^{\prime}}{\partial y^{\prime 2}}\right)-\delta \beta^{2} F^{\prime} \exp \left(\frac{\beta\left(T^{\prime}-1\right)}{1+\alpha_{h}\left(T^{\prime}-1\right)}\right), \\
& \frac{\partial T^{\prime}}{\partial t^{\prime}}-2 y^{\prime} \frac{\partial T^{\prime}}{\partial y^{\prime}}=\left(\frac{\partial^{2} T^{\prime}}{\partial x^{\prime 2}}+\frac{\partial^{2} T^{\prime}}{\partial y^{\prime 2}}\right)+\delta \beta^{2} F^{\prime} \exp \left(\frac{\beta\left(T^{\prime}-1\right)}{1+\alpha_{h}\left(T^{\prime}-1\right)}\right),
\end{aligned}
$$

where $\alpha_{h}=\left(T_{b}-T_{0}\right) / T_{b}$. Finally, removing the primes we have the differential equations

$$
\begin{aligned}
& \frac{\partial F}{\partial t}-2 y \frac{\partial F}{\partial y}=\frac{1}{\mathrm{Le}}\left(\frac{\partial^{2} F}{\partial x^{2}}+\frac{\partial^{2} F}{\partial y^{2}}\right)-\delta \beta^{2} F T^{\beta} \\
& \frac{\partial T}{\partial t}-2 y \frac{\partial T}{\partial y}=\left(\frac{\partial^{2} T}{\partial x^{2}}+\frac{\partial^{2} T}{\partial y^{2}}\right)+\delta \beta^{2} F T^{\beta}
\end{aligned}
$$

in which the Lewis number Le $=\mathrm{D}_{\mathrm{T}} / \mathrm{D}_{\mathrm{F}}, \alpha_{h}$ has been approximated by 1 and $\exp (\beta(T-1))$ by $T^{\beta}[5]$. Dirichlet, cold boundary conditions, namely $T=0$ and $F=1$, are imposed at $y= \pm Y_{m}$ and Neumann, independent of $x$ boundary conditions $\frac{\partial F}{\partial x}=0$ and $\frac{\partial T}{\partial x}=0$ are imposed at $x= \pm X_{m}$. In all the calculations we used the Zeldovich number $\beta=10$.

These equations have the cold solution $F=1, T=0$ for all time but for certain combinations of Le and $\delta$ they also have a stationary solution independent of $x$, namely the one dimensional flame in the burner described above. 
For a given Lewis number, there is a minimum Damköhler number (that is a maximum strain rate) for which combustion can take place. This minimum Damköhler number is called the quenching Damköhler number. When the Lewis number is significantly less than 1, then close to the quenching Damköhler number we find there are cellular flames. Thus we also find isolated and multiple stationary flame tube solutions of equations (3) and (4) for certain combinations of Lewis and Damköhler numbers.

To study drifting flame tubes, we first compute an isolated, stationary flame tube in a rectangular region in $x$ and $y$. We then place two flame tubes close together and carry out a time integration of the time dependent equations (3) and (4).

\section{Numerical method and grid definition}

The burning solutions of equations (3) and (4) are symmetric with respect to $y$. Thus solutions of these equations were computed in the computational region $-X_{m} \leq x \leq+X_{m}$ and $0 \leq y \leq Y_{m}$ subject to the boundary conditions

1. $T=0$ and $F=1$ on $y=Y_{m}$,

2. $\frac{\partial F}{\partial x}=0$ and $\frac{\partial T}{\partial x}=0$ on $x=-X_{m}$ and $x=X_{m}$,

3. symmetry conditions $\frac{\partial F}{\partial y}=0$ and $\frac{\partial T}{\partial y}=0$ on $y=0$.

The values of $X_{m}$ and $Y_{m}$ were chosen to be sufficiently large so that the boundary has negligible effect on the combustion calculations.

For this two dimensional problem the far field effects are not great since $F$ and $T$ approach their far field values exponentially. Thus a large region exterior to the flame tube was not necessary. At Lewis number $=0.4$ flame tubes exist for Damköhler numbers in the range, $0.011<\delta<0.026$. 


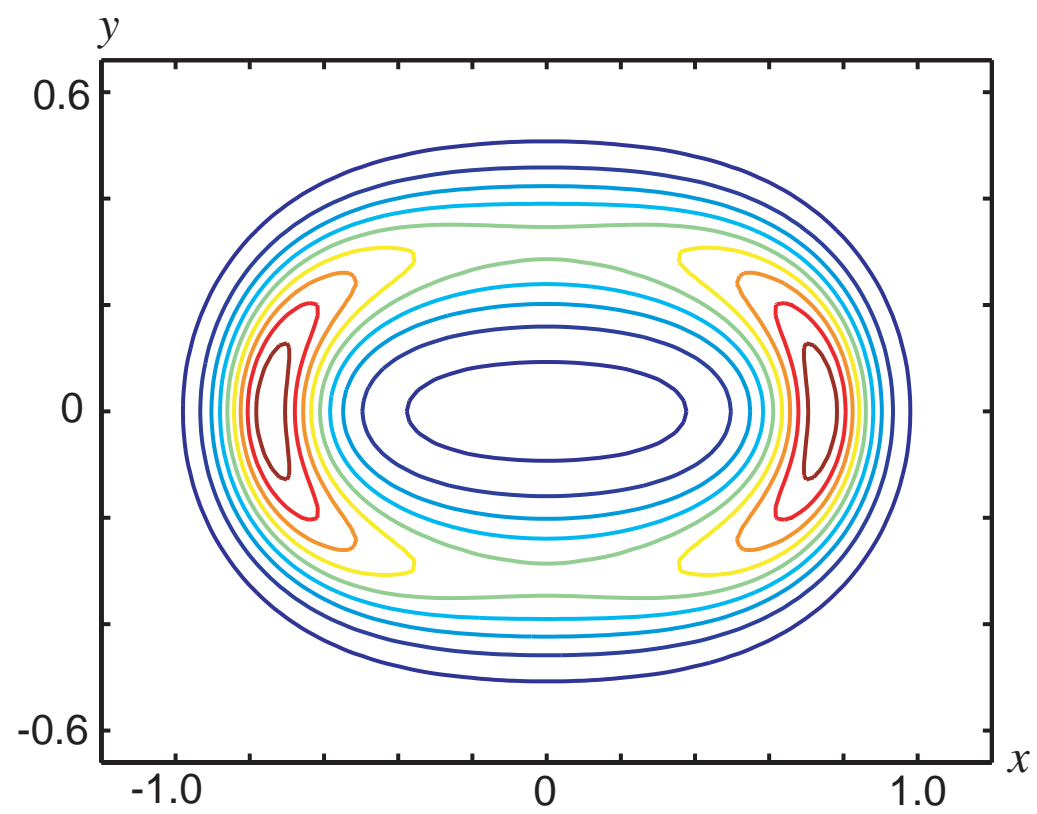

FiguRE 2: Rate of reaction contours for the stationary, premixed flame tube, Le $0.4, \delta=0.02, \beta=10$.

Choosing $\delta=0.02$ the flame tube was located in $-0.74<x<0.74$ and $-0.32<y<0.32$, see Figure 2. The boundary of the flame tube was taken to be the position of the maximum rate of reaction.

The computational domain chosen had values $X_{m}=20$ and $Y_{m}=5$, the larger value for $X_{m}$ being chosen to allow for flame tubes to drift. The grid used was uniform in the $x$ direction with $h_{x}=0.02$ and was uniform for $0 \leq y \leq 0.5$, with $h_{y}=0.02$. It was non-uniform for $0.5 \leq y \leq 5.0$ with $h=h_{y}^{(i)}$ for $i=1,2, \ldots, 25$ with $h_{y}^{(1)}=h_{y}$ and $h_{y}^{(i)}=\lambda+h_{y}^{(i-1)}, \lambda=0.01333$. Thus we used a $2000 \times 50$ rectangular grid. 
The stationary solution was computed by approximating the stationary form of equations (3) and (4) using a Galerkin finite element method with bilinear quadrilateral elements. The discrete equations were solved by Newton's method. The time dependent equations were also approximated by the Galerkin finite element method and the discrete system of first order ordinary differential equations were integrated using a very simple Euler code.

\section{Numerical results for two flame tubes}

To model the effect of two flame tubes, 900 grid lines parallel to the $y$ axis were removed from the left of the grid $(x<0)$ and an equal number added on the right of the grid. The initial solution for the time integration was the stationary solution at the remaining grid points augmented with a cold solution $(F=1, T=0)$ at the new grid points. The Neumann boundary condition on the left of the grid now acted as a symmetry boundary condition between two flame tubes whereas the Neumann boundary condition on the right represented the far field condition for large $x$. It was far enough from the flame tube to have negligible effect on its behaviour. A time integration was then performed.

We observed that the temperature at the mid point between the flame tubes initially increased while the flame tubes shrank in size and began to drift apart. Once this initial phase had passed, the integration settled down the flame tubes drifted apart with:

1. a speed that fairly rapidly approached, but did not reach, zero, see Figure 3;

2. the temperature at the mid point between the flame tubes decreasing as the tubes became further apart; 


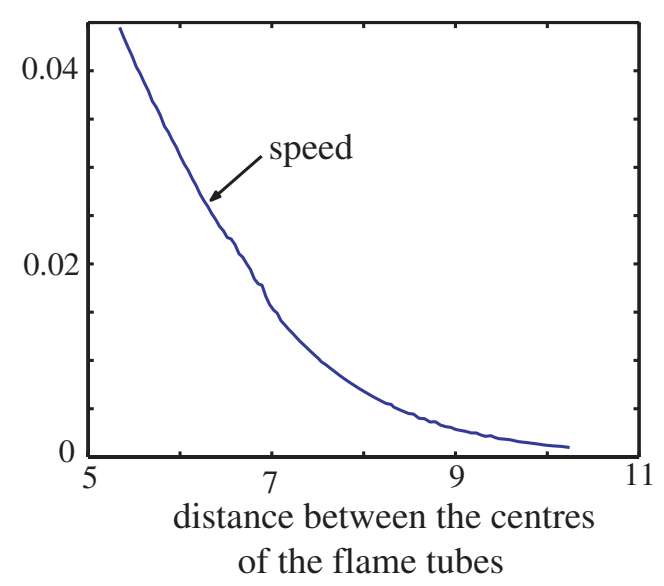

Figure 3: The velocity of the drifting flame tubes plotted against separation.

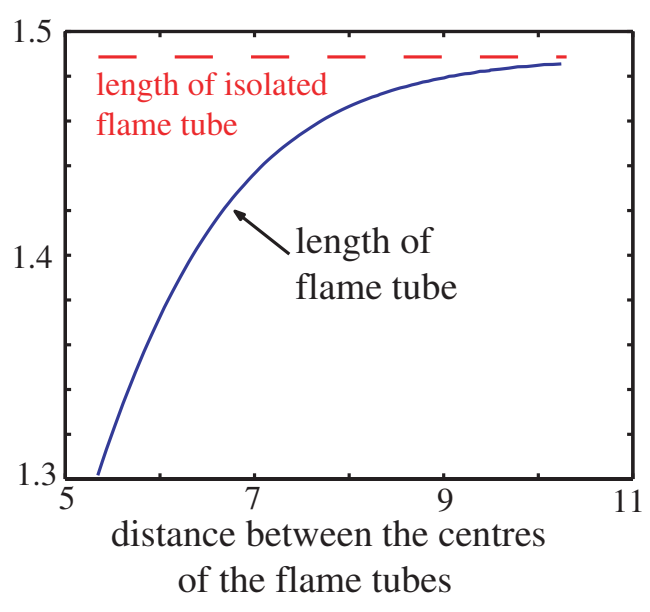

Figure 4: The length of the drifting flame tubes plotted against separation.

3. the size of the flame tube increasing and approaching the size of the stationary flame tube, see Figure 4 which shows how the length of the flame tube in the $x$ direction approached the length of the stationary flame tube.

Indeed, summing up these results, the two flame tubes behaved exactly as expected with the tubes moving into the fresh fuel mixture rather than competing with each other for the fuel mixture between the the two tubes. Moreover, as the flame tubes moved further apart their solutions were converging to that of the isolated flame tube. 


\section{Conclusion}

The numerical solutions presented here verified the expected drifting apart of flame tubes into regions of greater fuel concentration. It proved disappointing that we were unable to obtain similar results using one step chemistry to model flame balls drifting apart. These results will be described in a second paper together with a more sophisticated representation of the chemistry that does give flame balls moving apart.

Acknowledgements The authors acknowledge helpful conversations with John Dold and the financial support of the UNSW at ADFA and the University of Manchester.

\section{References}

[1] R. Daou, J. Daou and J. W. Dold, The effect of heat loss on a flame edge in a non-premixed counterflow. Combustion Theory and Modelling 7 (2003) 221-242. doi:10.1088/1364-7830/7/2/302 C619

[2] R. Daou, J. Daou and J. W. Dold, Effects of volumetric heat loss on triple flame propagation. Proc Combustion Institute 29 (2002) 1559-1564. doi:10.1016/S1540-7489(02)80191-2 C619

[3] C. Kaiser, J-B. Liu and P. D. Ronney, Diffusive-thermal instability of counterflow flames at low Lewis numbers. AAIA Paper No 2000-0576. Presented at 38th AIAA Aerospace Science Meeting, Reno (2000). http://pdf.aiaa.org/preview/2000/PV2000_576.pdf C619

[4] P. D. Ronney, M. S. Wu, H. G. Pearlman and K. J. Weiland, Experimental studies of premixed flame balls in space: preliminary results of STS-83. AIAA Journal 36 (1998) 1361-1368. 
http://pdf .aiaa.org/jaPreview/AIAAJ/1998/PVJAIMP553.pdf C619

[5] R. W. Thatcher and J. W. Dold, Edges of flames that do not exist: flame edge dynamics in a non-premixed counterflow. Combustion Theory and Modelling 4 (2000) 435-457. doi:10.1088/1364-7830/4/4/304 C619, C622 


\section{Author addresses}

1. R. W. Thatcher, School of Mathematics, University of Manchester, M60 1QD, UK

mailto:R.W.Thatcher@manchester.ac.uk

2. R. O. Weber, PEMS, UNSW at ADFA, Canberra 2600, Australia mailto:r.weber@adfa.edu.au 